insight into incidents, made them feel safer and prepared for the day, played a part in reducing restrictive practice, and empowered staff from all professional backgrounds by giving them a voice. Low or late participation, cancellation of $\mathrm{SH}$ because of clinical activity, and vague questions in the meeting template were identified as barriers in implementation.

Conclusion. Acute psychiatric wards regularly face challenges of high clinical activity, low staffing levels, bed pressures, and highrisk patient cohorts. SH contributed to reducing restrictive practice and creating a safer and more positive work environment. It is important to ensure SH are taking place daily, using an appropriate template to guide staff who may be new to facilitating. Accordingly, the impact on restrictive practice, patient care and staff wellbeing can be sustained long-term.

\section{Assessing DNA rates for new referrals in older adults}

\section{Aamina Cheema ${ }^{1 \star}$, Nasir Pasha ${ }^{2}$ and Giorgi Gurieli ${ }^{3}$}

${ }^{1}$ Little Bromwich Center, Neuropsychiatry - The Barberry Center;

${ }^{2}$ Little Bromwich Center - Older Adults CMHT and ${ }^{3}$ CT3 BSMHFT ${ }^{*}$ Corresponding author.

doi: 10.1192/bjo.2021.486

Aims. To assess DNA rates for initial assessment medical appointments offered for new referrals within an Older Adults CMHT.

To establish any correlation between waiting time and DNA rates.

To establish if the initial appointments offered were in keeping with the National guidelines (18 weeks) and our local Trust policy (1-4 weeks).

Background. In the Uk 15\% of adults 60 and above suffer from a mental disorder. Despite the increasing mental health burden, analysis indicate that a quarter of mental health trust received less investment from 2017 to 2018. Financial pressures have also increased appointment waiting time. The NHS has stated that by 2023 there will be a 4 -week waiting time for older adult mental health services. Current national guidelines state that initial referrals should be seen within 18 weeks.

Method. This is a retrospective audit looking at all first time referrals to an Older Adult CMHT in East Birmingham. 110 patients were included in this audit. Factors recorded included age, gender, reason for referral, waiting time for appointment, and whether this complies with guidelines.

Electronic patients' notes (RIO) were used for data collection. Result. Out of 110 new referrals 11 were not offered any appointments. Out of the remaining 99, 13 cancelled and 8 did not attend.

In total, 78 attended the initial appointment offered, out of which 77 were seen within 18 weeks as per national guidelines. 43 patients were seen within the 4 -week period (trust policy). 1 patient was offered an appointment at 19 weeks and 3 days from the referral date. The patients who did not attend their appointments were followed up except for one, to find out the reasons of the DNA. This included 2 (physically unwell), 1 (unaware of appointment), 1 (refused), 1 (forgot), 1 (couldn't get to clinic), 1 (asthma attack). Another appointment was offered to those who could attend.

Conclusion. There was no significant correlation between a longer waiting time and an increased DNA rate for first appointments. Even though the time for an initial appointment was within the NHS guidelines, only $56 \%$ of the appointments met our Trust's policy of a 4 week wait.
When discussing the results with the relevant team it was clear that a number of factors affected the waiting time including: number of available clinicians and a large catchment area.

Phew! time to focus on physical health and wellbeing: improving the assessment and management of physical health in an early intervention in psychosis service

\section{Bethany Cole*, Emma Bailey and Liz Ewins}

Avon and Wiltshire Mental Health Partnership NHS Trust ${ }^{*}$ Corresponding author.

doi: 10.1192/bjo.2021.487

Aims. NICE guidelines recommend that patients under Early Intervention (EI) in Psychosis Services have systematic monitoring and intervention of cardiometabolic risk factors. We undertook a Quality Improvement Project (QIP) in the Bath and North East Somerset (BaNES) EI Team to improve rates of compliance with national guidelines. We aimed to increase the percentage of service users with a physical health assessment documented in the past 12 months. Other aims included improving monitoring of physical health parameters in those taking antipsychotic medication and increasing the delivery of interventions for abnormal results.

Background. The most common cause of premature mortality in people who experience psychosis and schizophrenia is cardiovascular disease. The 'Standards for Early Intervention in Psychosis Service' states that patients should be offered personalised healthy lifestyle interventions, including advice on diet, physical activity, and access to smoking cessation services. Physical health should be monitored at least annually, with more frequent assessments if antipsychotic medication is prescribed.

Method. We identified seven key factors for improving physical health: Body Mass Index (BMI), Blood Pressure, Glucose Regulation, Blood Lipids, Smoking, Alcohol and Illicit drug use. Baseline compliance and intervention rates were measured in March 2019. Six 'Plan, Do, Study, Act' Cycles were completed over the following ten months. Examples of the changes made included: a new online diary and whiteboard, abbreviation of the assessment form, teaching for the EI team, and a new weekly 'Physical Health and Wellbeing' (PHeW) Clinic. This clinic involved phlebotomy, discussions around lifestyle choices, review of medication side effects, and neurological examination.

We measured the compliance with guidelines each month and the total number of interventions delivered at threemonthly intervals. We collected qualitative feedback on these changes in team meetings and with written questionnaires (including feedback from patients).

Result. Documentation of all key factors doubled from $30.2 \%$ at baseline to $63.3 \%$ in January 2020 . The total number of interventions for raised BMI and lipid levels also increased. Feedback from staff and patients was positive. The clinic helped start conversations with patients about lifestyle choices, prompting improvements in weight, physical activity, lipid levels, and alcohol intake. Patient awareness and ownership over their physical health also improved.

Conclusion. This project utilised multiple strategies to reduce health complications for BaNES EI service users. A structural change in the assessment and management of physical health proved to be an effective and sustainable solution to optimise the health and wellbeing of this patient group. 